\title{
Aportes del Análisis de Redes Sociales a la Gestión de Estrategias de Cooperación Empresarial'
}

\author{
Andrés Matta², Universidad Nacional de Córdoba (Argentina) - Universidad \\ Católica de Córdoba (España)
}

\section{Resumen}

El incremento de políticas y programas orientados a fomentar la cooperación empresarial contrasta con la escasez de evaluaciones que reflejen sus efectos en términos no económicos. En parte esto se debe a que no se han desarrollado suficientemente los modelos teórico-metodológicos para estudiar los efectos sociales de estas iniciativas.

Este artículo presenta un modelo de análisis elaborado con aportes del ARS, para estudiar la estructura de relaciones que emerge en el complejo de industrias electrónicas e informáticas de la ciudad de Córdoba (Argentina), durante su participación en una política de apoyo a la articulación productiva. Para esto se sistematiza un conjunto de indicadores a nivel de la escala, profundidad, eficacia y sostenibilidad de los efectos del programa.

Si bien se trata de un estudio de caso de carácter fundamentalmente descriptivo y predictivo, permite arribar a algunas conclusiones sobre el potencial de los programas para incrementar la frecuencia de las acciones cooperativas en mayor medida que su nivel de compromiso y la dificultad para modificar estructuras sociales preexistentes, en particular cuando se trata de redes lideradas por asociaciones empresarias sectoriales.

De este modo, se espera que los resultados de este trabajo pueden ser de utilidad para quienes diseñan, gestionan y evalúan procesos de articulación productiva y las diversas estructuras que de éstos se generan (grupos de empresas, redes, clusters, entre otros).

Palabras clave: Cooperación empresarial, Articulación productiva, capital social, indicadores.

\begin{abstract}
Increasing policies and programs to promote business cooperation contrasts with the lack of evaluations that reflect their effects in non-economic terms. In part this is because theoretical and methodological models to study the social impact of these initiatives are not developed enough.

This paper presents an analysis model developed with ARS, to study the structure of relationships that emerge in the electronic and computer industrie's complex in

\footnotetext{
${ }^{1}$ Este artículo es parte de la Tesis de Doctorado en Ciencias Económicas. Un análisis más exhaustivo puede encontrarse en el libro "Desarrollo de cadenas productivas, clusters y redes empresariales" coordinado por Felix Mitnik (Coord). Fondo Multilateral de Inversiones, Banco Interamericano de Desarrollo. Agencia de Desarrollo de la Ciudad de Córdoba (Argentina) (2011). Durante 2012 el FOMIN ha seleccionado este caso para realizar una nueva evaluación y construir herramientas aplicables a sus programas. Agradezco a estas instituciones y a las asociaciones CIIECCA y CCT sin las cuales este estudio no hubiera sido posible.

2 Universidad Nacional de Córdoba; Instituto de Administración; Facultad de Ciencias Económicas. Universidad Católica de Córdoba; Facultad de Ciencias Económicas y de Administración. amatta@arnet.com.ar.
} 
the city of Cordoba (Argentina), during a policy of "productive articulation". For this aim, we present a set of indicators to reflect the scale, depth, efficiency and sustainability of the program's effects.

Although this is a case study largely descriptive and predictive, we reach some conclusions about the potential of programs to increase the frequency of cooperative actions more than their level of commitment and the difficulty to change preexisting social structures, particularly in networks led by business associations.

Thus, it is expected that the results of this work can be useful for those who design, manage and evaluate productive articulation processes and the structures that they generate (groups of companies, networks, clusters, etc.).

Keywords: Business cooperation - productive articulation- social capital-indicators

\section{Introducción. Las estructuras sociales y la sostenibilidad de los procesos de integración}

En las últimas décadas y promovidos por diversos organismos internacionales se han desarrollado en América Latina numerosas políticas y programas orientados a incentivar la articulación productiva mediante el incremento de la cooperación entre empresas y el crecimiento del capital social. Su supuesto fundamental es que las acciones conjuntas entre firmas, o entre éstas y las organizaciones del sector público y la sociedad civil permiten mejorar la competitividad empresarial logrando resultados superiores a los obtenidos individualmente (efecto al que se denomina como eficiencia colectiva).

No obstante la centralidad que se le otorga a la cooperación interorganizacional en los objetivos de estos programas, es frecuente que a la hora de su evaluación, se valore solamente la utilidad individual de las estrategias conjuntas, cuanto mucho limitadas a generar "economías de escala". Al ser cada empresa particular la unidad de análisis, se concibe al proceso social que se fomenta como una "caja negra" o como una mera "suma aritmética" de organizaciones. La limitación de este enfoque tiene dos consecuencias: por una parte no permite comprender acabadamente de qué forma se llega a los resultados económicos en una particular trama social; por otro lado, obstruye la visión sobre la sostenibilidad de los lazos de cooperación fomentados y apoyados durante los procesos de integración. Así, desde el punto de vista conceptual y metodológico, muchos de estos programas acaban por no diferir sustancialmente de aquellos que incentivan a empresas que operan de manera aislada.

Desde una perspectiva alternativa, es posible afirmar que es imprescindible evaluar los efectos sociales de las políticas de integración productiva, ya que su éxito y sostenibilidad depende en buena medida de mejorar la calidad de las relaciones de 
cooperación interorganizacional. Si esta mejora no se produce, una vez finalizada la intervención y agotados los incentivos económicos coyunturales, puede descubrirse con desazón que -aún con buenos resultados económicos globales- se ha originado una situación estructuralmente inferior a la encontrada antes del programa. Esto ha sucedido por ejemplo en algunas políticas públicas donde los actores se asocian para cumplir con requisitos de accesibilidad pero luego no pueden sostener sus vínculos por falta de un apoyo específico o simplemente porque dichas relaciones no tenían el fundamento social adecuado (Etchegorry et al, 2009).

Con el objeto de enriquecer los modelos de monitoreo y evaluación de este tipo de programas y visibilizar sus resultados sociales, se han explorado los aportes del ARS a partir de un estudio de caso que analiza la trama empresarial del complejo industrial electrónico-informático de la ciudad de Córdoba (Argentina) durante la ejecución de una política de integración productiva. Si bien se trata de un estudio fundamentalmente descriptivo, originado en un enfoque constructivista, el trabajo realizado permite arribar a un conjunto de conclusiones útiles para el diseño, ejecución y evaluación de iniciativas similares.

\section{Marco conceptual. Cooperación, redes sociales y capital social}

Aunque no se profundizarán aquí los aspectos teóricos en toda su necesaria complejidad -a fin de priorizar el análisis de los datos- es preciso explicitar algunos de sus principales supuestos conceptuales.

En primer lugar, cabe señalar, que esta investigación sigue la línea de aquellos estudios que consideran que las prácticas económicas están inmersas en estructuras sociales (Polanyi, 1947; Granovetter, 1995; Uzzi, 1997; Bourdieu, 2001). Para aproximarse al fenómeno específico de la cooperación entre empresas se han escogido y privilegiado, entre otras nociones habitualmente utilizadas, los conceptos de "red social" y "capital social". Ambas concepciones, son ricas desde el punto de vista heurístico pero no están exentas de polémica por la multiplicidad de sus abordajes teóricos y metodológicos. Aún así, la mayor parte de las vertientes intelectuales consideran que las redes sociales son una forma de capital social, suceptible de ser utilizado por los actores en la consecución de sus fines e intereses.

En el caso de las redes sociales, se ha optado aquí por una definición "minimalista", considerándolas como conjuntos de relaciones (denominados lazos) entre agentes sociales individuales o colectivos (denominados nodos), que configuran una estructura dada por la cantidad y la calidad de los vínculos que la conforman. Esta 
definición es similar a la utilizada por otros autores (Aldrich y Zimmer, 1986) y en realidad es apenas un punto de partida para caracterizar un fenómeno que puede ser analizado desde diversas perspectivas.

Esta definición es además funcional a los efectos del estudio realizado, dado que permite reconstruir sobre ella las distintas formas de relación que pueden darse entre las empresas, sobre todo si se tiene en cuenta que las prácticas de cooperación o de acción colectiva suelen tener formas que no se adaptan exactamente ni a la jerarquización propia de las organizaciones burocráticas, ni tampoco al tipo propio de las relaciones de mercado (Adler y Kwon, 2002). Tomando como unidad básica de análisis la noción de "vínculo" o "relación", pueden construirse así los diferentes tipos de redes reflejando de manera más acabada un fenómeno que se caracteriza fundamentalmente por su espontaneidad, flexibilidad y variabilidad ${ }^{3}$.

Vale la pena aclarar que, al escoger este enfoque, este estudio no utiliza la noción de red en tanto forma específica de organización, sino como un sustrato de las prácticas económicas que pueden generar diversas formas de coordinación y de organización industrial (clusters, redes, cadenas productivas, complejos, etc. $)^{4}$. Como señalan Laumann, Marsden y Prensky (1983) se puede decir que el análisis de redes permite cierta flexibilidad al investigador al no imponer a priori asunciones sobre la agrupación de los actores, por lo cual, sea que se analice a un grupo de firmas, un sistema, o una red de redes interactuantes siempre es posible costruir indicadores mensurables a partir de considerarlos como una red. En la misma línea, el ARS no será considerado aquí como una teoría o "paradigma" al estilo kuhniano, sino como un conjunto de instrumentos que pueden ser utilizados en el marco de distintas teorías y propósitos (Baranger, 2004) y, particularmente, en el estudio de las prácticas de cooperación entre las empresas.

Asimismo, debe aclararse que lo que se denomina aquí como "relaciones", subsume al menos dos acepciones que desde el punto de vista sociológico podrían ser discriminadas. Una relación social puede ser entendida como referencia, si el análisis se centra en los agentes al estilo weberiano, considerando a la relación como un producto o proyección individual y un correlato de la acción social (en este

\footnotetext{
${ }^{3}$ Entre otros textos que pueden ilustrar a esta corriente de estudio de las redes sociales en el campo económico puede consultarse: Aldrich, H.,et al,. 1986; Baum, J.A.C.,et al.,2000 ;Birley, S., 1990; Casanueva Rocha C. 2003; Cross, R. et al. 2002; Granovetter, 1973 y 1995; Gulati, R. et al.1999, Hakansson, L., et al 1993; Johannisson, B. 2000; Johannison, B. et al 2000; Lin, N. 1999; Milward, B. et al 1998; Premaratne, 2002; Szarka, J. 1990; Veciana, J.M. et al 1996.

${ }^{4}$ No es posible aquí profundizar en el "caos semántico" que existe ante la proliferación de conceptos aparentemente similares como "cadena productiva", "cluster", "distrito empresarial" (Cfr.Matta, 2012).
} 
caso de acción colectiva $)^{5}$. Una relación social puede también concebirse como un vínculo o ligamen, lo que equivale a decir que no es sólo una proyección subjetiva de los agentes sino una realidad capaz de generar condicionamientos recíprocos en tanto estructuras a nivel psíquico y social. Considerar esta perspectiva para analizar las relaciones de cooperación implica enriquecer la visión puramente referencial para considerar que estas relaciones constituyen o son parte de estructuras con una densidad y autonomía mayores que un mero acontecimiento probabilísticamente esperado por los sujetos.

En el enfoque referencial, muchas veces lo que se denomina como relación, no es sino un contacto puntual generado a partir de una acción mientras que en el enfoque vincular se trata de un fenómeno de mayor densidad y persistencia. Sin desconocer esta precisión, en este estudio se considera ambas dimensiones como resultado de diferentes enfoques metodológicos y no como una inevitable alternativa ontológica. Una relación de cooperación puede ser una mera referencia si se analiza desde la subjetividad de los agentes (que queda particularmente expuesta cuando no existe reciprocidad) y al mismo tiempo un vínculo capaz de generar estructuras que exceden a la intencionalidad y pueden observarse tanto de manera objetiva como interiorizados en los agentes.

En cuanto a la noción de "capital social" también pueden reconocerse distintas tradiciones teóricas desde el "constructivismo estructuralista" (Bourdieu, 1980) o el "interaccionismo" (Coleman, 1990; Burt 1992, 2000), que aún con diferencias, coinciden en destacar su impacto en las prácticas económicas. Esta noción introduce la posibilidad de considerar a las relaciones de cooperación y las redes como una forma de capital en tanto éste puede acumularse, conservarse, apropiarse y convertirse en otras especies. A partir de los desarrollos iniciales, distintos estudios han procurado distinguir dentro del concepto algunas dimensiones que aquí se considerarán, tales como la relacional (centrada en la cantidad y calidad de las relaciones de los agentes), la estructural (que analiza las posiciones de los agentes en la trama social), la institucional (que valora las normas y las instituciones sociales), la basada en los "recursos" (que analiza el potencial de las redes para el acceso a distintas formas de capital) (Castro, 2005).

\footnotetext{
${ }^{5}$ El concepto y los problemas que conlleva defiinir la cooperación como "acción colectiva" han sido analizados desde diferentes perspectivas que en este caso omitiremos. Pueden verse por ejemplo los interesantes trabajos de Tuomela (2000). Además del enfoque de redes sociales, la cuestión de la cooperación entre empresas en el marco de las teorías inter-organizacionales también se halla en pleno desarrollo. Entre otros textos consultados puede verse: Ariño, A. et al 2001; Contractor, F.J. et al 1988; Doz, Y.L. 1996; Doz, Y.L., et al 1986; Lee, C., et al. 2001; Mohr, J., et al 1994; Parker, A. et al 2001; Sivadas E. et al 1998; Smith, J. et al. 1995; Steensma, H. K. et al 2000; Todeva, E. et al 2001; Tsai, W. 2000; Uzzi, B. 1996 y 1997.
} 
Tanto las nociones de "red social" como de "capital social" permitirán realizar un análisis en el cual los resultados económicos aparecerán claramente como imbricados en las relaciones de confianza, la existencia de lazos primarios, la presencia de antecedentes históricos negativos o de conflictos, así como diversas formas de poder y de distribución del trabajo.

Finalmente, si bien el estudio se centra en un momento específico del tiempo, se analizarán algunos aspectos vinculados con la evolución de la trama social en la medida que esto es importante para comprender los efectos de las políticas de integración productiva. Ciertas limitaciones del objeto de estudio y de los propios objetivos impiden utilizar los modelos dinámicos de reciente desarrollo como SIENA (Snijders, 2010), pero no obstante se comparan algunos indicadores básicos en dos períodos, prestando atención a fenómenos como la centralidad o los conflictos. En redes de cooperación estas variables permiten monitorear los riesgos potenciales que corre una estructura, sobre todo cuando los conflictos son de carácter "nuclear" y no "periféricos" (Labianca et al, 1998). Si bien en algunos casos los conflictos pueden estimular la creatividad o la productividad (Pelled et al, 1999), en el caso de las redes de cooperación, y sobre todo si éstas se basan en acuerdos informales, ciertos conflictos originados en comportamientos oportunistas pueden tener consecuencias estructurales afectando la confianza general (Williamson, 1995). Esto se ve agravado además cuando los conflictos no son diádicos y entre organizaciones marginales sino que pertenecen a los centros estructurales de una red (Scott, 2000).

\section{Metodología y fuente de datos}

El modelo desarrollado para monitorear y evaluar las estrategias interorganizacionales, surge de un estudio de caso realizado en el complejo electrónico-informático de la Ciudad de Córdoba, constituido mayoritariamente por micro, pequeñas y medianas empresas que se hallaban asociadas a dos instituciones: la Cámara de Industrias Informáticas, Electrónicas y de Comunicaciones del Centro de Argentina (CIIECCA) y al Cluster Córdoba Technology (CCT). Este complejo fue apoyado por una política público-privada denominada Programa de Desarrollo de Cadenas Productivas durante aproximadamente tres años entre 2004 y 2007.

Las actividades conjuntas promovidas por el Programa podrían clasificarse en cinco tipologías: i) las vinculadas con el desarrollo de centros de producción o de servicios (se destaca un Centro de Montaje de componentes y un Centro de Abastecimiento del Sector Electrónico); ii) la certificación de calidad y acreditación 
de madurez (en el sector informático); iii) el desarrollo de competencias laborales; iv) las actividades de fortalecimiento institucional (planificaciones estratégicas, contratación de gerentes o coordinadores para las asociaciones empresarias; apoyo a la modificación de normas legales); v) las destinadas al posicionamiento social y comercial a través de la participación en ferias y eventos.

Para analizar sus efectos, se realizaron encuestas semiestructuradas a los propietarios/directivos de las firmas en dos años consecutivos. En el primer año (2005) se obtuvieron datos de 50 empresas participantes del Programa y de 14 no participantes. Esto implica que se relevó a un $85 \%$ de las firmas participantes y a un $73 \%$ de las empresas de todo el complejo. Si bien el estudio se centra en los datos de esta primera encuesta, en un segundo año, se realizó una onda complementaria que abarcó a un $70 \%$ de las empresas participantes y que permitió analizar la evolución de la red "tratada" por el Programa. Si bien no se llegó a la totalidad de las firmas, la magnitud del relevamiento y algunas indagaciones posteriores, permiten considerar aquí que los resultados de los análisis reflejan la situación del complejo durante los años estudiados, en particular del núcleo de firmas de capitales nacionales más importantes y con mayor centralidad.

Apelando a la importancia de la triangulación metodológica (Denzin, 1970), se realizaron además entrevistas a agentes claves, observación participante en reuniones y eventos, y se presentaron los resultados preliminares a las empresas para obtener información cualitativa.

Vale la pena mencionar que uno de los supuestos más importantes de este estudio es que en las empresas de menor tamaño, como las que aquí se analizan, es posible considerar que existe una estrecha relación ( $y$ en general un solapamiento) entre el empresario y la empresa/organización. Por cierto las organizaciones pueden ser vistas como un "campo" en el que cada miembro actua según sus estrategias (Bourdieu, 2001) o como un conjunto complejo de relaciones contractuales (Jensen y Meckling, 1976) pero en las empresas pequeñas, en las que propiedad y dirección se asimilan en una misma persona, es posible pensar en que la información aportada por los empresarios refleja las relaciones de la propia organización ${ }^{6}$.

A fin de sistematizar algunos de los principales aportes obtenidos, se ha adaptado una de las categorizaciones más utilizadas en la evaluación de programas y

\footnotetext{
${ }^{6}$ Un estudio de N. Berti (2006), que da cuenta de la historia del sector en Córdoba da pie para suponer que esta hipótesis sería acertada.
} 
políticas de desarrollo de MiPyMEs (McVay, 2000). Este esquema tiene en cuenta las siguientes dimensiones: i) la cobertura o extensión, que describe la escala alcanzada por la intervención de un programa o política ii) el alcance o profundidad, en algunos casos llamado "acceso", que complementa las medidas de escala con una perspectiva sobre la capacidad de incidir en ciertos segmentos específicos iii) la efectividad, que analiza el cumplimiento de los objetivos previstos en una intervención iv) la sostenibilidad de los efectos de la intervención, que analiza en qué medida los cambios generados por el programa pueden persistir en el tiempo sin su intervención. No detallaremos en este caso la sostenibilidad económicofinanciera ni la sostenibilidad institucional, que son objeto de otros estudios. Tampoco se analizará separadamente el impacto de la intervención, ya que si se utilizan las dimensiones precedentes, éste puede resumirse a partir de la efectividad y la sostenibilidad. Un estudio de impacto más profundo debería incluir mediciones muy posteriores a la intervención y la utilización (siempre que fuera posible) de grupos de control.

Para analizar estos aspectos se construyeron diferentes redes que recogen información de diversos aspectos del fenómeno de la cooperación empresarial ${ }^{7}$ : el grado de conocimiento previo y de comunicación (tanto en frecuencia como en calidad), la acción colectiva (cooperación) tanto en cantidad como en intensidad (compromiso) de las actividades conjuntas, los resultados de la acción en términos de eficacia y eficiencia económica pero también en lo referido al impacto en la trama social. Asimismo se han relevado diferentes variables complementarias para caracterizar las relaciones (competencia, liderazgo, relaciones primarias y secundarias, participación en las decisiones, conflictividad, etc.) y las empresas (tamaño, antigüedad, sector, etc.).

Estas redes reflejan además intercambios de distinta índole: económicos, en la medida que todas las actividades implicaban erogaciones por parte de los empresarios y en algunos casos la asunción colectiva de créditos; de información, indagando particularmente el flujo de datos considerados sensibles o estratégicos; de amistad, en tanto las relaciones entre empresarios facilitan los vínculos organizacionales.

\footnotetext{
7 Para los distintos análisis y gráficos se ha escogido en este caso Ucinet (Borgatti, et al, 2002) Visone (Brandes et al., 2006). KeyPlayer (Borgatti y Dreyfus).
} 
Si bien este artículo recoge aspectos más bien descriptivos de la investigación, también se presentan análisis predictivos a partir del uso de métodos usuales en ARS como el Quadratic Assignment Procedure (QAP). A partir de este procedimiento se extraen diversos índices como los de Jaccard y Pearson, utilizados para correlacionar matrices binarias 0 valuadas. Estos indicadores tienen una interpretación similar al índice de correlación utilizado para variables atributivas, aunque se trata de variantes del método bootstrap basado en permutaciones. Algo similar sucede con las regresiones entre matrices y sus R2 (Simpson, 2001).

\section{Sistematización de resultados}

\subsection{Cobertura o extensión de las relaciones entre las empresas}

Una primera observación de la estructura de la red conformada por las empresas que declararon haber cooperado en el Año 1 permite constatar que no se trata de una red totalmente integrada sino de dos sub-redes claramente diferenciadas, con centros estructurales más densos (Scott, 2000) y un grupo de firmas que ofician como "cutpoints" (Gráfico 1). Entre éstas últimas se destacan cuatro empresas que pertenecen a las dos asociaciones empresarias (en color verde) mientras que en la periferia y como firmas aisladas se ubican las firmas "no participantes" del Programa (en color blanco).

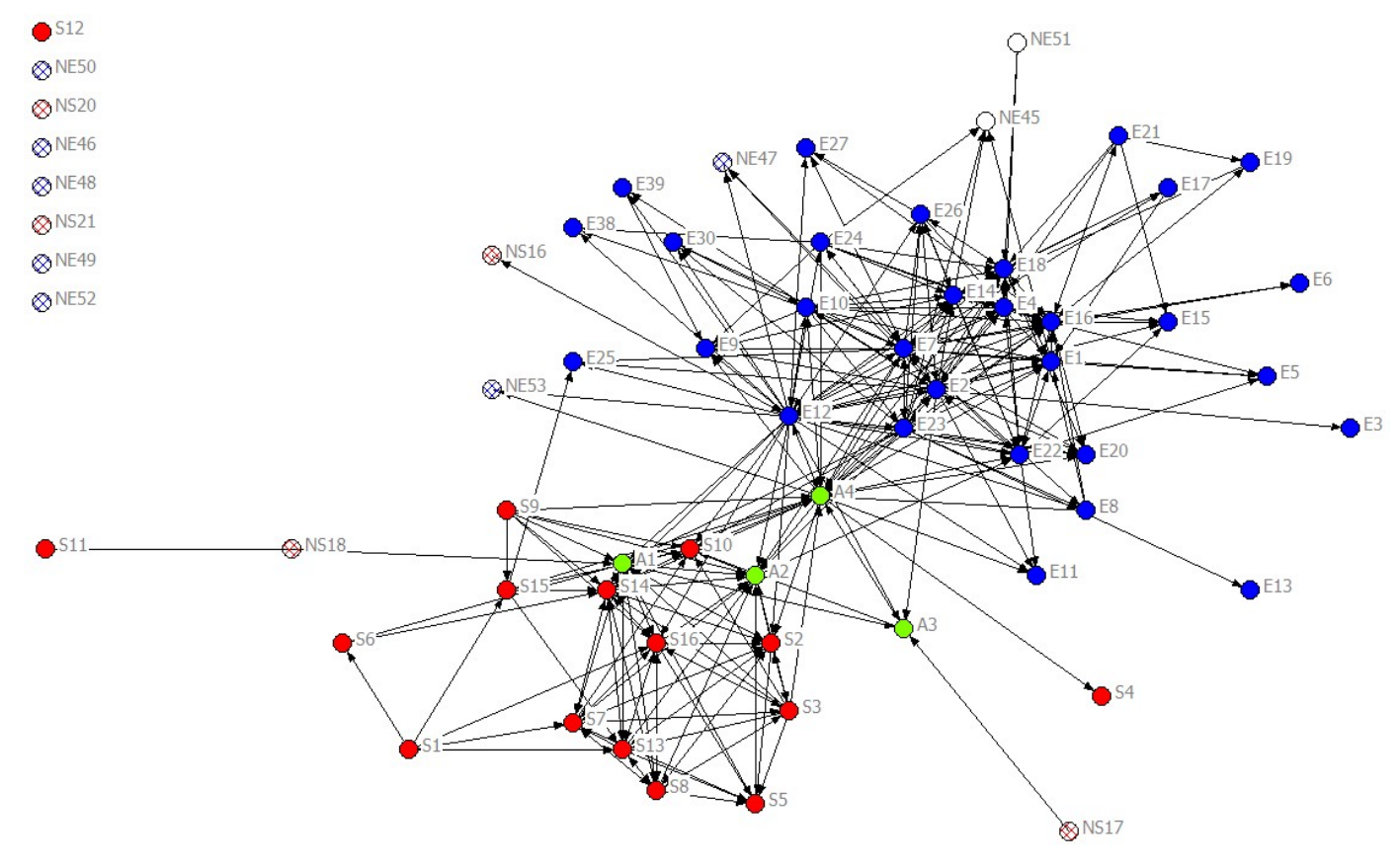

S: empresas CCT E: empresas CIIECCA NS y NE: no participantes del programa

Gráfico 1. Red de Cooperación Directa (Año 1). 
En lo que respecta a la cantidad de relaciones observada en la trama de empresas, la Tabla 1 permite observar de manera sintética algunos de los indicadores aportados por este estudio para esta dimensión. En la misma se observan los promedios y densidades de la red completa, de las dos "subredes" conformadas por las empresas del CCT (mayormente industrias informáticas), de CIIECCA (mayoritariamente industrias electrónicas) y de las empresas que pertenecen a ambas asociaciones. Esta primera dimensión permite visibilizar las características globales de la red total y las diferencias entre los dos sectores industriales.

Respecto al conocimiento y la comunicación, en promedio las empresas conocían en el primer año a 21 miembros de la red (un tercio de la misma), comunicándose con 8 de ellos aunque sólo con 2 intercambiando información considerada sensible.

Respecto a la cooperación, cada firma realizó en promedio una actividad conjunta, cooperando directamente con 5 empresas y con otras 7 de manera indirecta, lo que significa que se ha actuado colectivamente con más de la midad de las firmas conocidas. Un $35 \%$ de las relaciones de cooperación son múltiples, es decir, con más de una actividad común.

En general puede decirse que es baja la competencia entre las firmas (cada empresario sólo percibe a dos pares como competidores) y en promedio existen relaciones de coopetencia (Brandenburger y Nalebuff, 1996; Lado, Boyd y Hanlon, 1997; Gnyawali y Madhavan, 2001) con la mitad de los competidores.

Por su parte, además de las 5 empresas en promedio con las que se cooperó de modo directo, en el futuro se estaría dispuesto a cooperar o se ha planificado efectivamente cooperar con 4 empresas y profundizar los vínculos con 3 al tiempo que no se estaría dispuesto a cooperar en promedio con 2 empresas ${ }^{8}$.

\begin{tabular}{lcccc}
\hline & CCT & CIIECCA & AMBAS & TOTAL \\
Cuántas empresas conoce & 20 & 22 & 29 & 21 \\
Con cuántas empresas se comunicó & 7 & 8 & 12 & 8 \\
$\begin{array}{l}\text { Con cuántas empresas comunicó información } \\
\text { confidencial }\end{array}$ & 3 & 2 & 6 & 2 \\
$\begin{array}{l}\text { Cuántas actividades conjuntas realizó } \\
\text { Con cuántas empresas cooperó de manera directa }\end{array}$ & 1,2 & 0,6 & 5,3 & 1 \\
$\begin{array}{l}\text { Con cuántas empresas cooperó de manera indirecta } \\
\text { \% promedio relaciones múltiples }\end{array}$ & 11 & 5 & 10 & 5 \\
A cuántas empresas considera competidoras & $39 \%$ & $30 \%$ & $63 \%$ & $35 \%$ \\
\hline
\end{tabular}

\footnotetext{
8 Estas 5 empresas con las que en promedio cooperan estas firmas, recuerdan a la "five key" de Johannisson (2002), quien señalaba que este era el número de relaciones claves de un emprendedor para iniciar su empresa.
} 


\begin{tabular}{lcccc}
\hline Con cuántas empresas existió coopetencia & 3 & 0,3 & 2,25 & 1,28 \\
Con cuántas empresas planifica cooperar & 4 & 3 & 11 & 4 \\
$\begin{array}{l}\text { Con cuántas empresas estaría dispuesto a cooperar } \\
\begin{array}{l}\text { Con cuántas empresas no cooperaría por existir } \\
\text { conflictos }\end{array}\end{array}$ & 3 & 3 & 9 & 4 \\
Con cuántas empresas produndizaría su cooperación & 3 & 1 & 0,3 & 1 \\
\hline
\end{tabular}

Tabla 1. Indicadores generales de cobertura. Relaciones promedio según sub-red (Año 1)

Más allá de estos datos generales, la Tabla 1 permite observar diferencias entre las "subredes" destacándose claramente el rol de las empresas que ofician de "cutpoints" y pertenecen a ambas asociaciones empresarias. Además se observa que las firmas informáticas poseen mayores niveles de competencia y de conflicto a pesar de lo cual han realizado más actividades conjuntas que las empresas electrónicas aunque con mayor cantidad de relaciones indirectas ${ }^{9}$.

Un análisis más minucioso de estos datos, permite observar una aparente inconsistencia ya que se habría cooperado con más firmas que con las que las que existió comunicación. Una serie de datos complementarios a los expuestos permite considerar que no se trata de un típico problema ocasionado por deficiencias en la memoria de los empresarios encuestados (McCarty et al., 2007; Molina, 2005) sino de un fenómeno relevante para comprender lo que sucede en este tipo de redes al que hemos denominado como "cooperación indirecta".

La "cooperación indirecta" implica que las empresas participan de actividades colectivas como parte de un conjunto vasto y difuso de acciones "intermediadas" por otras empresas o por entidades tales como las asociaciones empresarias. Para reforzar esta tesis, se debe considerar que en muchos casos las firmas refirieron haber cooperado con "empresas de CIIECCA y CCT", no pudiendo identificar empresas individuales.

También es relevante mencionar que según los registros internos del Programa existió un nivel de cooperación tres veces mayor que el que reconocieron las empresas encuestadas, lo que en este caso indica que si no se realiza un relevamiento exhaustivo, los ejecutores de las políticas pueden sobreestimar la

\footnotetext{
${ }^{9}$ En general los niveles de coopetencia encontrados son bajos, entre otras cosas porque también lo son los niveles de competencia, siendo mayores en el sector de industrias de software donde más allá de la competencia actual, todas son "competidoras potenciales" (la elevada especialización de las firmas hace que se concentren en nichos de mercado diferentes).
} 
colaboración de empresas que se registran formalmente en las actividades pero luego no participan ${ }^{10}$.

En síntesis, hasta el momento el estudio revelaría que el Programa ha propiciado mayor cantidad de relaciones de cooperación entre las firmas participantes que las que se dan entre las no participantes. Al no contarse con una línea de base no es posible decir con certeza en qué medida este fenómeno se ha dado por influencia del Programa o por un proceso de autoselección, pero es posible confirmar esta hipótesis al analizar otros indicadores (por ejemplo las actividades planificadas para el año posterior a la finalización del Programa también son mayores).

También revela que estas relaciones se distribuyen de modo desigual, con mayores valores para las empresas que pertenecen simultáneamente al CCT y a CIIECCA, lo que revela el rol gravitante de la pertenencia a las asociaciones empresarias.

\subsection{El alcance o profundidad del capital social relacional}

La segunda dimensión utilizada para analizar los efectos del Programa permite visibilizar cuál es la intensidad de las relaciones de cooperación y en qué medida se ha alcanzado a diferentes segmentos de empresas.

La intensidad se ha estudiado en función de la frecuencia de las actividades conjuntas pero también del nivel de compromiso de las relaciones entre firmas. Para esto se ha elaborado un indicador que recoge tres aspectos de las actividades conjuntas: sus costos en términos económicos y de inversión en tiempo, su nivel de riesgo y su duración. Esto permite no ponderar de igual modo la realización de una actividad esporádica y de bajo costo (como por ejemplo asistir conjuntamente a una jornada de capacitación), que otra que tiene mayores implicancias (como la adquisición y utilización conjunta de bienes de capital).

La Tabla 2 permite observar que en el primer año relevado las empresas del CCT son las que han realizado o participado en promedio de mayor cantidad de actividades, aunque con niveles de compromiso similar a las firmas de CIIECCA. En el segundo año no sólo disminuye esta cantidad de actividades sensiblemente en el CCT $(-55 \%)$ sino que las empresas de la CIIECCA aumentan la cantidad de actividades $(40 \%)$ y (levemente) su nivel de compromiso, lo que marca una diferencia de tendencia en la evolución de las relaciones entre ambas subredes.

\footnotetext{
10 Aunque se evita al lector los detalles, vale la pena mencionar que para construir las redes de cooperacion existen diferentes alternativas que el investigador debe evaluar: a partir de los registros internos del Programa; de las percepciones de las empresas ante la pregunta "con qué empresas cooperó"; de construir una "red de afiliación" a partir de las actividades en las que se dice participar.
} 


\begin{tabular}{|c|c|c|}
\hline & CCT & CIIECCA \\
\hline Proporción de socios participantes del programa & $65 \%$ & $72 \%$ \\
\hline $\begin{array}{l}\text { Cantidad de actividades realizadas por empresas de la sub-red } \\
\text { (Año 1) }\end{array}$ & 9 & 4 \\
\hline $\begin{array}{l}\text { Evolución de cantidad de actividades realizadas por empresas } \\
\text { de la sub' red }(\%)\end{array}$ & $-55 \%$ & $+40 \%$ \\
\hline Promedio de compromiso de actividades por empresa ( 0 a 3 ) & 2,08 & 2,10 \\
\hline Proporción de actividades de compromiso medio-alto (Año 1) & $22 \%$ & $20 \%$ \\
\hline Proporción de actividades de compromiso medio-alto (Año 2) & $25 \%$ & $29 \%$ \\
\hline $\begin{array}{l}\text { Participación de Micro y Pequeñas empresas en la red (según } \\
\text { cantidad de empleados) }\end{array}$ & $47 \%$ & $77 \%$ \\
\hline $\begin{array}{l}\text { Proporción de relaciones de las Micro y Pequeñas empresas } \\
\text { (según cantidad de empleados) }\end{array}$ & $63 \%$ & $44 \%$ \\
\hline Proporción de relaciones asimétricas (red confidencial) & $60 \%$ & $58 \%$ \\
\hline Promedio de participación en actividades (0-5) & 3,95 & 3,55 \\
\hline
\end{tabular}

Tabla 2. Indicadores generales de alcance y profundidad.

En cuanto a la capacidad del Programa para alcanzar a las empresas de menor tamaño, se observa una diferencia importante entre ambas sub-redes, ya que las micro y pequeñas empresas representan sólo un $47 \%$ en la red del CCT, pero acumulan el $63 \%$ del total de relaciones existentes mientras que en CIIECCA se da un fenómeno inverso ${ }^{11}$. Esta situación probablemente se deba a las particularidades propias de las empresas de cada sub-red, dado que en el CCT la red es más pequeña pero más homogénea en relación con el tamaño de las empresas.

Los indicadores de intensidad sugieren en general que el complejo electrónicoinformático de Córdoba posee una trama caracterizada en general por lazos débiles y dividida en dos sub-redes centralizadas por dos núcleos en los que sí existen lazos fuertes y relaciones de coopetencia. Esto puede observarse en la Tabla 3 donde se observa que los cliques centrales tienen diferencias significativas respecto a la periferia, no sólo en su cantidad de actividades y relaciones directas (grados) sino también en sus niveles de compromiso promedio. También se observa que son consideradas por sus pares como líderes de las actividades y que en algunos casos como en CIIECCA existe una mayor proporción de relaciones de amistad.

\begin{tabular}{lccc}
\hline & $\begin{array}{l}\text { Núcleo } \\
\text { CIIECCA }\end{array}$ & $\begin{array}{l}\text { Núcleo } \\
\text { CCT }\end{array}$ & Periféricas \\
\hline Cantidad actividades (primer año) & 4,00 & 7,10 & 3,11 \\
Cantidad de actividades (segundo año) & 4,45 & 2,90 & 1,85 \\
Cooperación directa (primer año) & 32,46 & 33,90 & 10,75 \\
\hline
\end{tabular}

\footnotetext{
${ }^{11}$ Cabe aclarar que en este caso se ha utilizado la clasificación por cantidad de empleados. Según su facturación, todas las empresas de la CIIECCA y más del $90 \%$ de las del CCT encuestadas son micro y pequeñas empresas.
} 


\begin{tabular}{lccc}
\hline \% Relaciones de cooperación directa & 19,84 & 21,90 & 5,97 \\
Nivel de compromiso promedio (0-3) & 2,02 & 2,09 & 1,80 \\
Nivel máximo de compromiso alcanzado (0-3) & 2,34 & 2,40 & 1,85 \\
Líderes (grado de entrada) & 3,23 &, 80 &, 34 \\
Relaciones de amistad (grado de salida) & 2,15 & 1,80 & 1,17 \\
Relaciones de amistad (grado de entrada) & 3,61 & 2,00 &, 65 \\
\hline
\end{tabular}

Tabla 3. Comparación de medias entre núcleos y periferia

En segundo lugar, se observa que estos núcleos centrales coinciden en ser en buena medida empresas de tamaño mediano y en muchos casos miembros de las comisiones directivas de las asociaciones empresarias. En la Tabla 4 se constata que es mayor el promedio de actividades conjuntas entre las empresas que forman parte de estas comisiones que fuera de ellas, un punto que tiene capital importancia a la hora de analizar la "gobernanza" de estas redes: todo indica que no es posible pensar en acciones de apoyo a la cooperación sin considerar el rol social estratégico de las instituciones empresariales.

Como señalan otros estudios, esto es particularmente relevante en este tipo de redes con lazos horizontales y débiles (denominadas "de aglomeración") (Casanueva Rocha, 2003). Este aspecto se refuerza al observar el posicionamiento y los resultados obtenidos por aquellas empresas que pertenecen a ambas asociaciones.

\begin{tabular}{lcc}
\hline & $\begin{array}{c}\text { Pertenecen } \\
\text { a comisiones }\end{array}$ & $\begin{array}{c}\text { No pertenecen } \\
\text { a comisiones }\end{array}$ \\
\hline Pertenecen a comisiones & 1,6 & 0,66 \\
No pertenecen a comisiones & 0,66 & 0,27 \\
\hline
\end{tabular}

Tabla 4. Promedio de actividades cruzadas según pertenencia de empresarios a comisiones directivas

También se ha constatado que si bien el Programa habría influido sobre todo en la cantidad de actividades realizadas, el "grado de compromiso" de las empresas no participantes es similar en promedio al de las participantes.

Finalmente, el estudio demuestra que existe una relación significativa entre el grado de conocimiento y comunicación y la cantidad promedio de actividades realizadas (Gráfico 2). En una relación entre dos empresas, el conocimiento y la intensidad de la comunicación están positivamente correlacionadas con la cantidad de actividades conjuntas. También son significativas aunque menores las relaciones con el nivel de compromiso de las actividades. La correlación es mayor si se la compara con el máximo nivel de compromiso al que ha llegado la relación. Algo similar sucede si se realiza el análisis tomando como unidades a las empresas: aquellas que más firmas conocen y con más frecuencia se comunican en promedio son también las que cooperan en mayor cantidad e intensidad de actividades. Si 
bien estos valores no indican relación de causalidad, es posible decir que la frecuencia de comunicación brinda una relevante aproximación a lo que sucede en la red de cooperación. Un mejor indicador es aún la existencia de comunicación de información sensible para las empresas, observando que el $91 \%$ de las empresas vinculadas por este tipo de intercambio han tenido relaciones de cooperación.

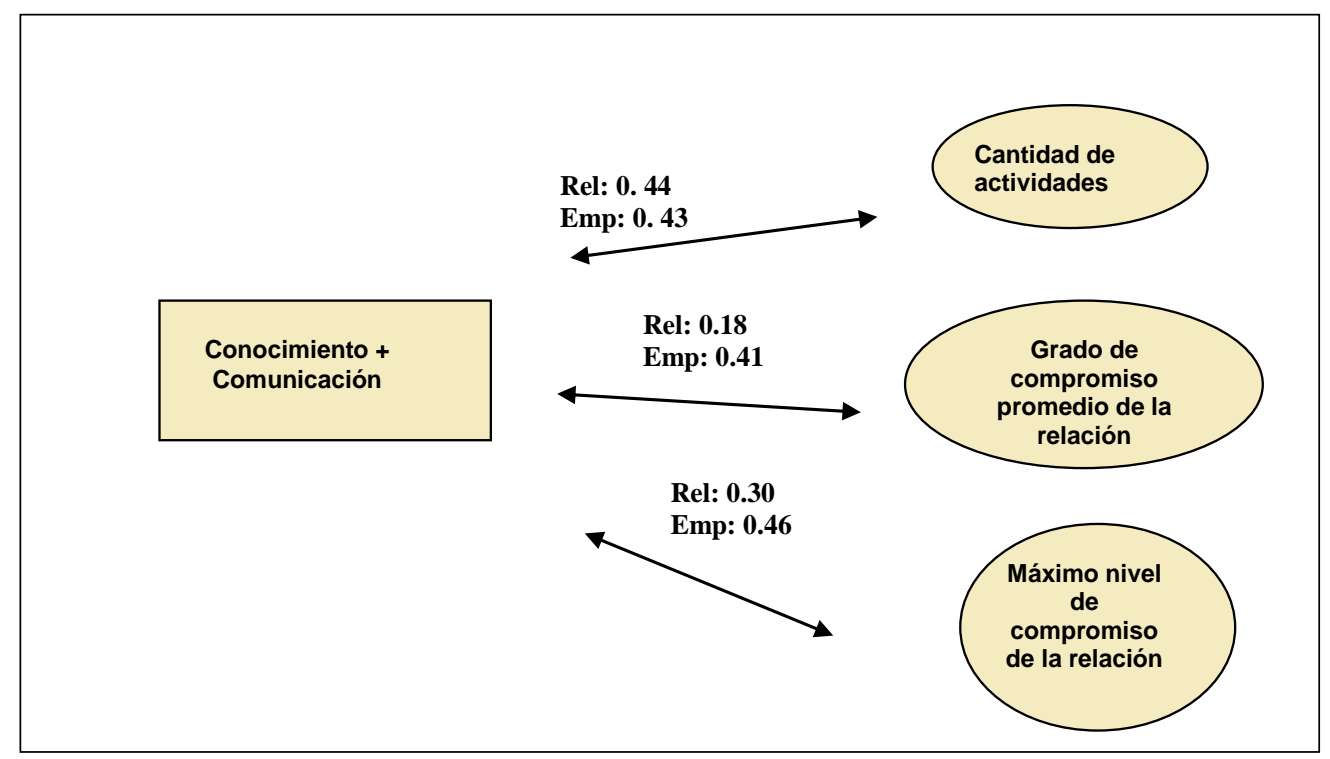

Gráfico 2. Correlaciones entre conocimiento/comunicación y relaciones de cooperación. Para las relaciones (Rel.) se utiliza indice de Pearson QAP. Para las Empresas (Emp.) Rho de Spearman. En todos los casos los valores son significativos.

\subsection{Eficacia y eficiencia colectiva}

Teniendo como trasfondo la discusión sobre cuál es el rol del capital social en los procesos que procuran aumentar la integración productiva, es relevante analizar la eficacia de esta estrategia para lograr un aumento de la cooperación y -por su intermedio- de la "eficiencia colectiva". Este concepto utilizado con frecuencia en la literatura sobre clusters (Pietrobelli y Rabellotti, 2006), podría ser definido desde algunas de las teorías del capital social como el aprovechamiento por parte de los individuos de su estrategia de relaciones y acciones colectivas. Si bien este enfoque permite observar los resultados económicos del capital social, no se analiza en esta etapa hasta qué punto existe un potencial conflicto entre este aprovechamiento y la sustentabilidad de las relaciones de cooperación.

Algunos autores (Kale et al., 2000) distinguen a los efectos de su análisis entre el capital social estructural y el individual. El primero podría resumirse como las ventajas de estar inserto en una red con una densidad y calidad de relaciones determinada. La segunda tiene que ver con la posición particular que cada empresa ocupa en dicha red y las ventajas obtenidas por el aprovechamiento de esa 
posición. Para analizar estos aspectos se estudiaron las variaciones en el período de referencia en relación con el nivel de facturación de las empresas y el empleo generado y se correlacionaron con las diferentes dimensiones de las redes ya analizadas para observar en qué medida se hallan vinculadas entre sí.

Antes de realizar este análisis debe señalarse que se trata de un complejo industrial de un dinamismo y resultados económicos extra-ordinarios en este período analizado. Los aumentos de la facturación de la Tabla 5 no son atribuibles solamente a la cooperación sino a factores exógenos vinculados con la coyuntura sectorial y macroeconómica y es baja la proporción de firmas que atribuye la mejora al Programa.

Un análisis desde el punto de vista del "capital social estructural" permite observar por su parte que las empresas que más han aprovechado su posición en la red son las empresas que pertenecen a ambas asociaciones empresarias. Todas las firmas en esta situación perciben que su facturación mejoró por la acción del Programa y el promedio de este incremento es mayor que para el resto de las firmas.

\begin{tabular}{|c|c|c|c|}
\hline & CCT & CIIECCA & AMBAS \\
\hline Aumento promedio de facturación en el útimo año & $66 \%$ & $58 \%$ & $51 \%$ \\
\hline Aumento promedio de facturación atribuido al programa & $7 \%$ & $12 \%$ & $40 \%$ \\
\hline $\begin{array}{l}\text { Proporción de empresas cuya facturación mejoró por el } \\
\text { programa }\end{array}$ & $10 \%$ & $\mathbf{2 1 \%}$ & $100 \%$ \\
\hline Aumento promedio de empleo atribuido al programa & $0 \%$ & $10 \%$ & - \\
\hline Aumento promedio de exportaciones * & $69 \%$ & $66 \%$ & $50 \%$ \\
\hline $\begin{array}{l}\text { Aumento promedio de exportaciones atribuido al } \\
\text { programa * }\end{array}$ & $8 \%$ & $7 \%$ & $50 \%$ \\
\hline Percepción de la relación costo-beneficio promedio (0 a 5) & 3,5 & 3,9 & 4 \\
\hline
\end{tabular}

Tabla 5. Indicadores generales de eficacia (Año 1)

Además de los resultados expuestos, desde el punto de vista del "capital social individual", el estudio revela que la mejora en el porcentaje de facturación de las empresas se relaciona directamente con la posición prominente de las empresas en las distintas redes analizadas (en función de su centralidad de grado). Esto significa que no se benefician todas las empresas que poseen más relaciones ni tampoco las de mayor tamaño, sino aquellas que tienen mayor poder y liderazgo. Entre las características de las empresas que son escogidas por las restantes para cooperar, se destaca su "capital comercial" (un posicionamiento superior en el mercado y mayor volumen de exportaciones), y su acumulación de mayor experiencia en actividades de cooperación (evidenciada por su participación en actividades de 
mayor compromiso y éxito). La posición de poder de estas empresas se ratifica por el hecho de que éstas son las que consideran que más participación han tenido en las decisiones de las actividades.

No obstante, si bien el tamaño de las empresas no garantiza automáticamente mejores resultados, esto no significa que el tamaño de las empresas no guarde relación con esta dimensión. Si tomamos el interés por cooperar como un indicador indirecto de los resultados obtenidos, la Tabla 6 muestra que la cantidad de relaciones de cooperación aumenta en función del tamaño de las firmas hasta que se llega a una suerte de "techo" en el que comienza a descender (o en este caso a desaparecer). El segmento compuesto por las empresas medianas, sin ser el segmento más numeroso, ha compartido mayor cantidad de actividades incluso con el resto de los segmentos. Una posible interpretación a este hecho es que las empresas más pequeñas, cooperan en menor medida y cuando lo hacen, es bajo su nivel de liderazgo, mientras que las firmas grandes en general tienden a trabajar de manera aislada (chi cuadrada 401,95 sig. 0,0007). Probablemente las empresas grandes no requieren de la cooperación con otras firmas, mientras que las pequeñas por su parte encuentran mayores barreras de entrada para generar este tipo de relaciones. Esto coincide en parte con lo observado en estudios como el Rabellotti y Schmitz (1999) quienes con una metodología diferente a la aquí utilizada) muestran que en las empresas de tamaño medio existe una asociación positiva entre la cooperación y el desempeño.

\begin{tabular}{lccccc}
\hline $\begin{array}{l}\text { Tipología y cantidad } \\
\text { de empresas }\end{array}$ & Micro & Pequeñas & Medianas & Grandes & Total \\
\hline Micro (15) & 30 & 69 & 99 & 0 & 198 \\
& $(15 \%)$ & $(35 \%)$ & $(50 \%)$ & $(0 \%)$ & $(100 \%)$ \\
Pequeñas (25) & 69 & 126 & 218 & 0 & 413 \\
& $(16 \%)$ & $(31 \%)$ & $(53 \%)$ & $(0 \%)$ & $(100 \%)$ \\
Medianas (21) & 99 & 218 & 274 & 0 & 591 \\
& $(12 \%)$ & $(42 \%)$ & $(46 \%)$ & $(0 \%)$ & $(100 \%)$ \\
Grandes (3) & 0 & 0 & 0 & 0 & 0 \\
& $(0 \%)$ & $(0 \%)$ & $(0 \%)$ & $(0 \%)$ & $(0 \%)$ \\
\hline
\end{tabular}

Tabla 6. Cantidad y densidad (\%)de relaciones de cooperación cruzadas por tamaño de firma (Año 1)

Siguiendo la concepción del capital social de P. Bourdieu (1980) y de N. Lin (1999), podemos considerar que el capital social no se define sólo por las redes de relaciones sino, sobre todo, por los recursos a los que es posible acceder gracias a estas redes. Además de las variaciones en la facturación, otra manera de observar cómo se ha dado este fenómeno en esta red es analizando la dirección de los flujos de información estratégica (a partir de la red de comunicación confidencial). Entre otros aspectos, puede estudiarse si la cooperación ha reforzado las asimetrías dadas por el tamaño de las empresas o ha favorecido intercambios de mayor 
horizontalidad. Este aspecto puede visualizarse claramente en el Gráfico 3 en el que pueden observarse los flujos de información considerada sensible, observándose la posición marginal de las firmas de menor tamaño y el rol prominente de las firmas medianas. Por otro lado, se advierte que entre las firmas que han recibido mayor cantidad de información y poseen mayor cantidad de relaciones simétricas, ocupan un lugar especial las empresas que conforman las comisiones directivas de las asociaciones empresarias (algo particularmente evidente en el CCT).

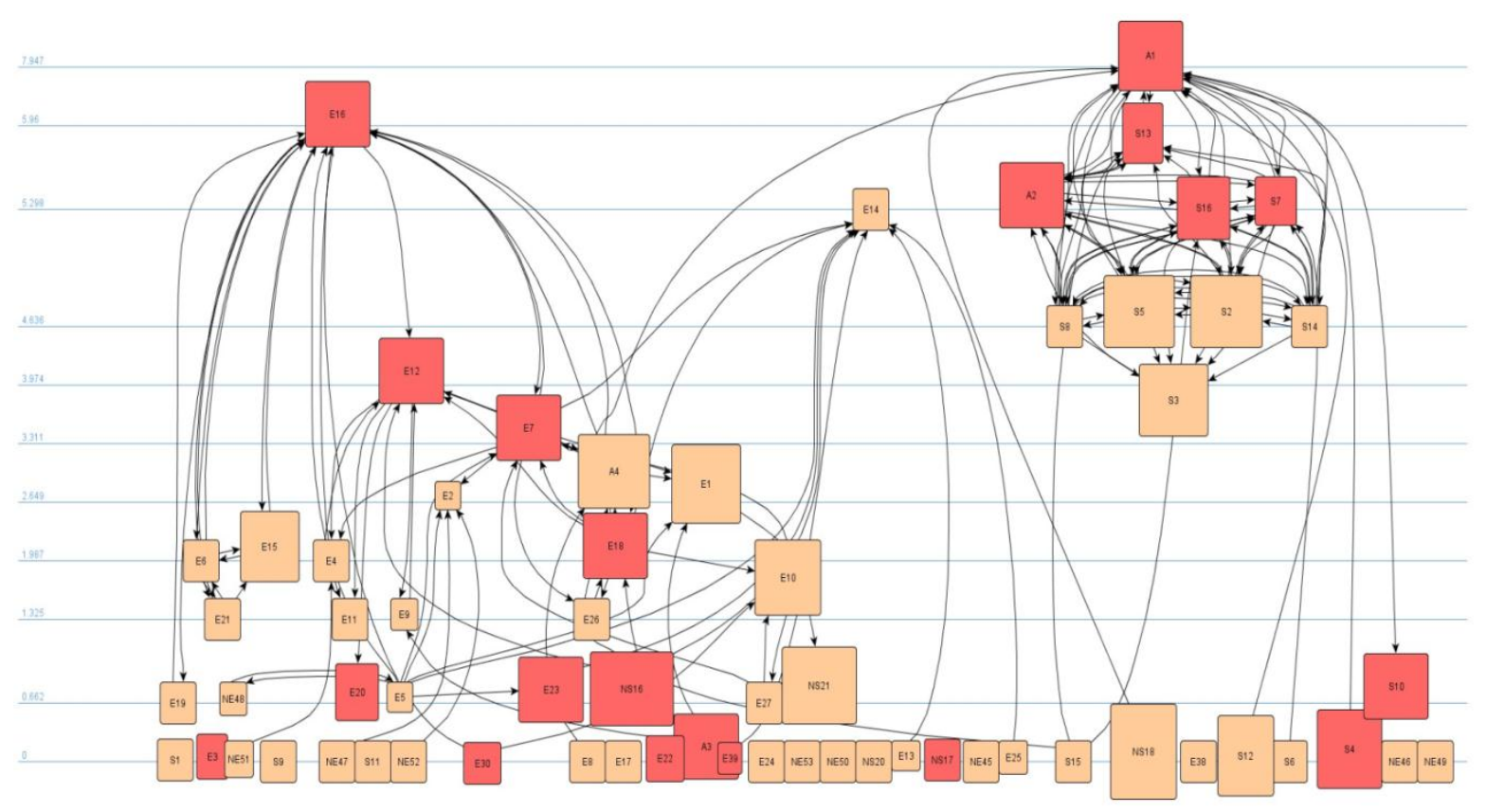

Elaboración propia. El tamaño de los nodos corresponde al tamaño de la empresa. El color más oscuro corresponde a las firmas pertenecientes a comisiones directivas de las asociaciones empresarias (Software Visone).

Gráfico 3. Flujo de información considerada sensible

\subsection{Sostenibilidad del capital social}

La sostenibilidad es uno de los objetivos más relevantes en todos los programas de apoyo a MiPyMEs desarrollados en las últimas décadas. Dejando de lado la sostenibilidad institucional, en este caso, el análisis se concentra en la sostenibilidad de los efectos del Programa en términos de capital social. Para ello se reconstruyeron dos dimensiones sobre el futuro de las redes: a) pragmática: hasta qué punto se logran mantener en el tiempo la cantidad y calidad de los vínculos entre las empresas b) actitudinal: en qué medida se mantiene o aumenta la predisposición a cooperar en el futuro. Para esto se construyeron tres redes que distinguen la existencia de planes de cooperación y las actitudes positivas hacia la cooperación futura. La comparación de las redes en ambos años de estudio confirman el carácter predictor de estas redes ya que se ha corroborado que en el 
$86 \%$ de los casos hubo relaciones de cooperación de acuerdo a lo planificado, en tanto que se cooperó en un $71 \%$ de las relaciones en las que existía una actitud positiva hacia la cooperación y en un $77 \%$ de las relaciones que se esperaba profundizar (en los casos donde coincidían los tres factores, se dio una coincidencia del $87 \%)$.

Sobre la relación entre lo decisional (planificado) y lo actitudinal (la predisposición a cooperar) se ha observado que las relaciones planificadas coinciden en un $44 \%$ con las relaciones de carácter hipotético en las que se desearía profundizar y un $31 \%$ con aquellas relaciones en las que se estaría dispuesto a cooperar. Esto quiere decir que existe una mayor predisposición a cooperar que lo que efectivamente se concreta, lo que abre para la gestión la posibilidad de continuar aumentando la cooperación, tanto en profundidad como en cantidad de nuevas empresas. Este dato podría ser además otro indicador de la magnitud de la cooperación indirecta ya que quiere decir que numerosas empresas planifican cooperar con otras con las que quizás no tienen siquiera una predisposición manifiesta a cooperar.

Como en las dimensiones anteriores, la Tabla 7 resume las principales variables relacionadas con la sostenibilidad de una trama de cooperación promovida por un programa o iniciativa. Estos datos indicarían una mejor evolución de la sub-red de las empresas electrónicas de CIIECCA, con un aumento en la cantidad de asociados y una menor densidad de conflictos, aunque se observa también un leve aumento de la centralización.

Como se ha dicho anteriormente, la pertenencia a las asociaciones empresarias ha sido clave en estas redes de cooperación y un programa de duración acotada, que pretenda la sustentabilidad de sus efectos en el capital social, debe cooperar con el fortalecimiento de las instituciones, sobre todo si estas coinciden con sus propósitos. Como ya se ha mencionado el estudio muestra un fortalecimiento de ambas instituciones, pero especialmente de una de ellas (CIIECCA) cuyo crecimiento en socios fue del $42 \%$ (incluyendo en esa cifra un flujo de empresas del CCT que se afiliaron en el segundo año del estudio) y que además aumentó la cantidad de actividades conjuntas y su grado de compromiso. 


\begin{tabular}{|c|c|c|}
\hline & CCT & CIIECCA \\
\hline Cantidad de empresas asociadas (Año 1) & 37 & 55 \\
\hline Evolución de cantidad de asociados (Año 2) & $+14 \%$ & $+42 \%$ \\
\hline $\begin{array}{l}\text { Cantidad promedio de empresas con las que no se cooperaría por } \\
\text { conflictos (Año 1) }\end{array}$ & 2,10 & 1,2 \\
\hline $\begin{array}{l}\text { Cantidad promedio de empresas con las que no se cooperaría por } \\
\text { conflictos (Año } 2 \text { ) }\end{array}$ & 2,46 & 1,2 \\
\hline Densidad de red de cooperación planificada (Año 1) & 0,14 & 0,06 \\
\hline Evolución de densidad de red de cooperación planificada (Año2) & $-70 \%$ & $0 \%$ \\
\hline Centralidad de grado red planificada 2005 (entrada-salida) & $79 \%-39 \%$ & $32 \%-18 \%$ \\
\hline $\begin{array}{l}\text { Centralidad de grado red planificada } 2006 \text { (entrada-salida) con } \\
\text { nuevas empresas }\end{array}$ & $30 \%-13 \%$ & $46 \%-22 \%$ \\
\hline$\%$ promedio de socios nuevos en segundo año & $10 \%$ & $20 \%$ \\
\hline $\begin{array}{l}\text { Nivel promedio de percepción sobre la existencia de socios } \\
\text { alternativos }(0 \text { a } 4)\end{array}$ & 2,63 & 2,68 \\
\hline
\end{tabular}

Tabla 7. Indicadores generales de sostenibilidad

Como se ve en el Gráfico 4, las variables que mejor predicen la cooperación en el futuro son la cantidad de actividades conjuntas y en menor medida el nivel de compromiso entendido como el nivel máximo de compromiso al que ha llegado la relación en alguna actividad. Entre las características de las actividades que parecen claves para asegurar la sustentabilidad, además del compromiso, se destaca el nivel de participación (incluso más que el nivel de compromiso) lo que significa que las empresas valoran particularmente este aspecto a la hora de decidir continuar cooperando. No obstante también existen relaciones positivas respecto a los resultados de las actividades, particularmente el grado de satisfacción y la mejor relación costo-beneficio percibida.

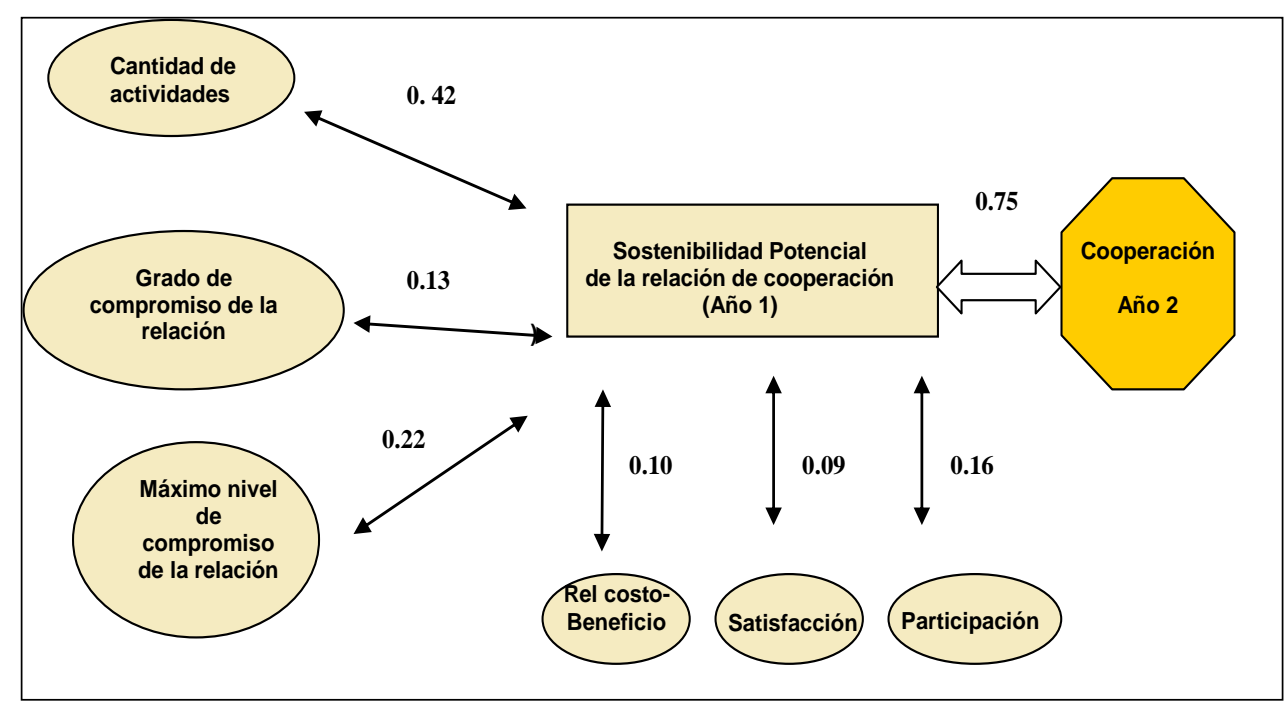

Gráfico 4. Factores de sostenibilidad potencial de las relaciones de cooperación (indice Pearson-QAP). 
Si en lugar de analizar la sostenibilidad potencial de la relación de cooperación examinamos como en el Gráfico 5, desde el punto de vista de las empresas, cuáles son los factores que aumentan la posibilidad de ser escogidas para cooperar, podemos observar nuevamente la importancia de los antecedentes (en cantidad de actividades y compromiso), y también atributos tales como el tamaño y su "capital comercial" mensurado por su capacidad exportadora.

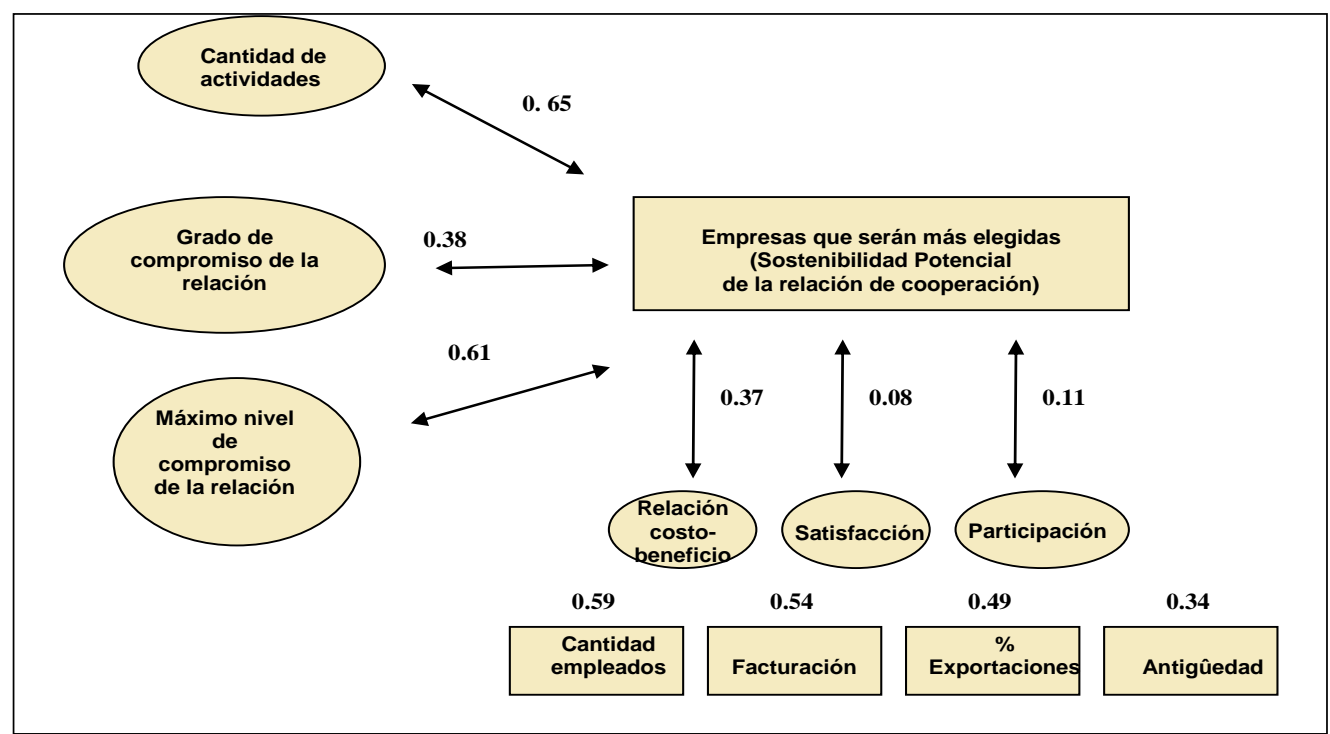

Gráfico 5. Factores de elegibilidad para la cooperación de empresas (Índices Rho de Spearman)

Entre los indicadores de potenciales factores de riesgo para la sostenibilidad se pueden mencionar por su parte cuatro. En primer lugar, el mayor nivel de competencia, que favorece la aparición de comportamientos individualistas en empresas con mayor volumen de capital y poder. En este caso, se evidencia a partir de la evolución de las densidades de las diferentes redes durante el período de referencia, que la CIIECCA mantiene su densidad interna, y aumenta a su tiempo la densidad de lazos con empresas del CCT, mientras que las empresas de esta última organización bajan su densidad interna. Esta situación puede verse en el Gráfico 6 que visibiliza la evolución de la comunicación de información sensible del primer y segundo año y revela las consecuencias de un "conflicto nuclear" ocurrido en el marco de esta sub-red que puede incluirse en este tipo de actitudes. 


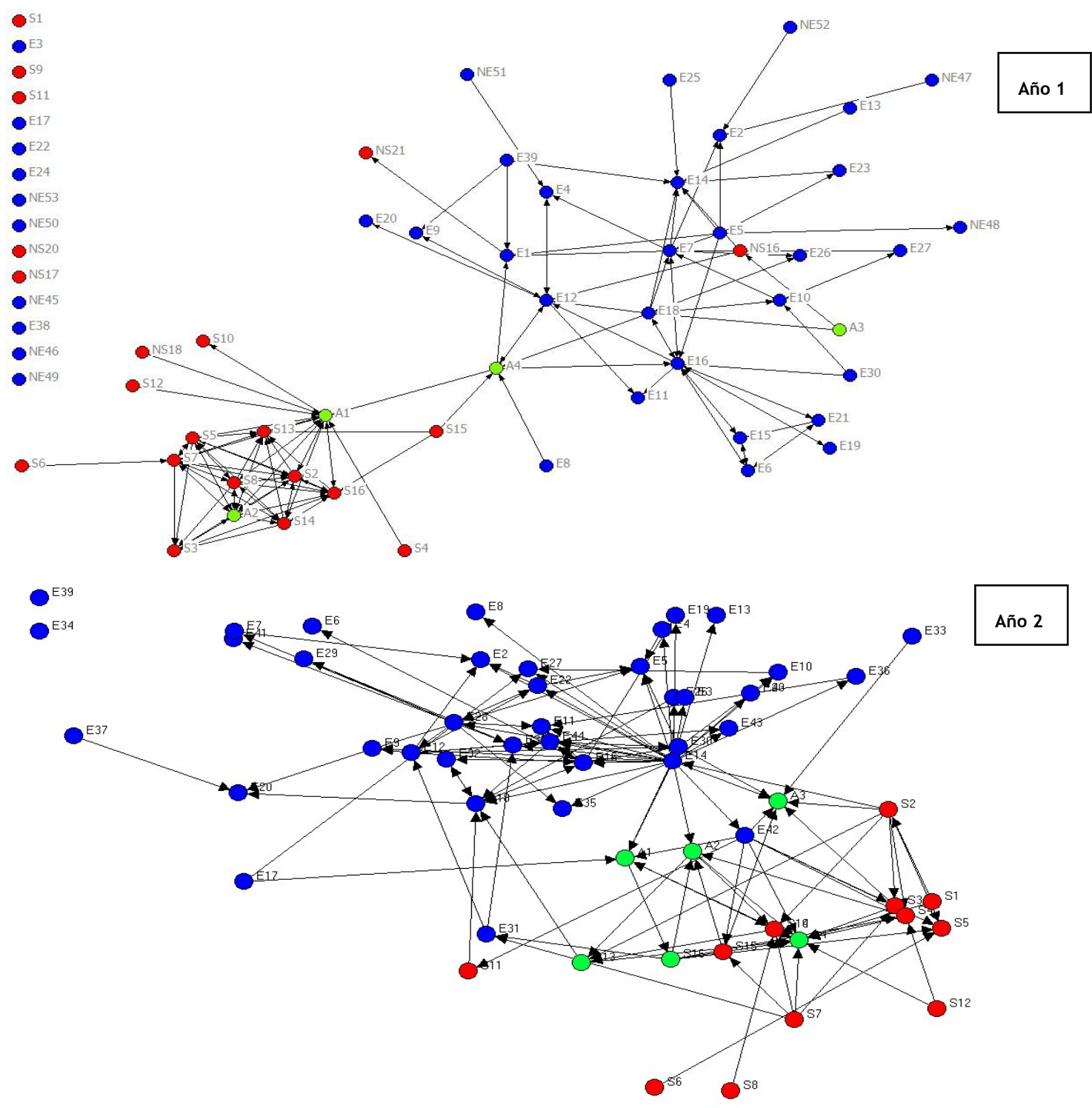

Gráfico 6. Evolución de red de comunicación confidencial

Un segundo factor de riesgo y que puede mensurarse a partir del ARS es el grado de centralización de la red. Si se presupone que la red es más vulnerable cuanto más dependiente sea de un conjunto reducido de empresas observamos que el cambio más notorio se da entre las empresas del CCT, con una muy alta centralización en el primer año y que -a causa del conflicto mencionado- reducen su grado de concentración a menos de la mitad al año siguiente. En cambio, en el caso de la CIIECCA y de la red total los valores se mantienen en niveles moderados (si tomamos las mismas empresas que en el primer año) o aumentan levemente (si incluimos las nuevas empresas), probablemente debido a la incorporación de mayor cantidad de empresas bajo el liderazgo del núcleo de firmas ya descripto anteriormente. Algo similar puede decirse respecto a la reducción de la "centralidad de intermediación" que desciende del 22\% al 15\%, debido fundamentalmente a 
que se han integrado mejor las dos redes y a que algunas empresas que pertenecían a ambas asociaciones perdieron su lugar prominente.

Un tercer factor es el nivel de conflictividad: del conjunto de la red construida se deriva una densidad de 0,1 con un $34 \%$ de las firmas que mencionaron algún tipo de conflicto (cada una no cooperaría con un promedio de 1,38 firmas lo que representa el $7 \%$ del conjunto de empresas que conoce). En el segundo año, estos valores aumentan ya que entre las mismas empresas el nivel de conflicto se eleva a una densidad de 0,34, (confirmando las consecuencias negativas de las acciones individualistas entre las empresas del sector software), sobre todo en el caso de las empresas que pertenecen a ambas asociaciones. Esta variable es relevante para evaluar la situación de la red. En este caso, como se trata de un sector relativamente joven y en expansión, no es extraño que los valores sean relativamente bajos.

El último factor a tener en cuenta, que se esboza a modo de hipótesis tiene que ver con la relación entre el tamaño de la red y la percepción por parte de los agentes sobre su nivel de dependencia y la existencia de potenciales socios alternativos. El supuesto básico de esta hipótesis es que es más difícil que una trama de relaciones se recupere de una situación de conflicto cuanto mayor sea la percepción de las empresas de que no existe la posibilidad de desarrollar relaciones alternativas. En este caso, la respuesta promedio de las empresas ha sido que "es posible reemplazar a algunas de las empresas" en cada una de las actividades realizadas. Probablemente, esta percepción, dependa también de otros factores como el nivel de conocimiento entre las empresas y la tipología de los vínculos que caracterizan a las relaciones en un sector. Al respecto, autores como Castells (1996) señalan que las nuevas empresas basadas en el "espíritu del informacionalismo" aprenden a vivir en una cultura de lo efímero, donde se construyen y se destruyen alianzas con cada decisión estratégica con la misma velocidad y fugacidad de las redes virtuales. Si bien las empresas estudiadas no parecen ajustarse totalmente a esta descripción, probablemente sean las empresas del CCT las que más respondan a este modelo.

En cuanto a cuál es el rol que ha tenido en el Programa en esta dimensión de análisis, las empresas participantes revelan mejores indicadores de cooperación en el futuro, y -como ya se ha visto- las relaciones que se conservan son aquellas en las que hay mayores actividades conjuntas. Como mencionamos anteriormente para el caso de la cooperación, al no contarse con datos de una línea de base y con información más completa sobre las empresas no participantes en el segundo año, 
no es posible comprobarlo con total exactitud pero en cambio puede observarse que un tercio de las relaciones que se conservarían al finalizar el programa son empresas que cooperaron en ambos años.

\section{Conclusiones}

A modo de cierre, y acorde con los objetivos propuestos, se presentan dos tipos de conclusiones a partir de los resultados empíricos y de los aprendizajes metodológicos con la finalidad de aportar al diseño de políticas públicas y privadas de integración productiva.

El estudio de este caso, indicaría que los instrumentos utilizados por el Programa permitieron incrementar la frecuencia de las acciones cooperativas a un nivel superior a las de las firmas no participantes, pero no tuvieron similar efecto en su compromiso promedio. No debe desmerecerse este resultado en la medida que un aumento en la cantidad de acciones incrementa la confianza, el aprendizaje y las competencias cooperativas, pero exceptuando algunos casos vinculados a la compra y uso colectivo de bienes de capital (en este caso presente en sólo una de las sub-redes), los programas de corto plazo corren el riesgo de generar acciones que luego no se sostienen en el tiempo. Esto podría revertirse generando estrategias incrementales, con incentivos adecuados para promover acciones paulatinas de mayor compromiso.

Paralelamente, se observa en este caso que los resultados económicos de la cooperación no han sido extraordinarios en el corto plazo. Es probable que esto sea frecuente en las etapas iniciales de un proceso cooperativo y por tanto, no se debe evaluar al Programa solamente por indicadores económicos sin considerar la tipología de las actividades (particularmente su horizonte temporal) y sin valorar qué condiciones sociales se han generado para que los resultados económicos se perciban en el futuro.

Desde el punto de vista estructural, los datos analizados aquí indican que el Programa reforzó o al menos no modificó ciertas estructuras sociales preexistentes: la separación de dos sectores industriales (electrónica e informática), el carácter conglomerado de las subredes con mayoría de lazos débiles, alta centralización y liderazgo de las asociaciones empresarias. Las indagaciones cualitativas indican que esto no fue un efecto previsto y que se realizaron acciones para minimizarlo. En función de esta experiencia, es altamente probable que este fenómeno se reitere en otros casos donde la política opere sobre la base de este tipo de redes y en cada caso se debe evaluar si este es un resultado aceptable. Esto es tanto más relevante 
cuando se comprueba que la política permite que los cutpoints, y los centros estructurales acumulen y concentren más poder (en términos de capital social) y aprovechen mejor sus beneficios. Para ello, además de estudiar las redes, se deben analizar al inicio aspectos vinculados a la historia, la cultura y las instituciones que serán determinantes en esta tendencia.

No se debe minimizar además la importancia de la participación de las firmas en las decisiones de cada actividad, ya que esta variable aparece como una de las relacionadas con la mayor disposición de las firmas a seguir cooperando en el futuro.

En el caso se pueden observar también las consecuencias de comportamientos oportunistas a partir de lo sucedido en una de las sub-redes. Estas conductas generan un mayor nivel de conflicto, alterando su densidad estructural y poniendo en riesgo su sostenibilidad. Si bien son ampliamente conocidos los beneficios de las relaciones de cooperación no formalizadas como alternativa a otras formas de gobernanza, probablemente los Programas deban brindar asistencia técnica para generar acuerdos y normas éticas compartidas que prevengan estas situaciones o establezcan mecanismos para su resolución. Además, este tipo de casos suele darse con mayor frecuencia en casos como este, donde se buscan resultados económicos de corto plazo en el marco de lazos sociales de corta trayectoria.

Finalmente, si bien el estudio permite observar que la existencia de lazos fuertes (si se los entiende en términos de relaciones comunitarias de amistad o parentesco) no es condición necesaria para que se generen relaciones de cooperación esta afirmación debe ser matizada. En primer lugar, los programas deben tener en cuenta las características del sector empresarial y de sus empresarios, ya que esto es más factible en sectores económicos relativamente recientes y en los cuales las prácticas económicas y comerciales están caracterizadas por un alto nivel de profesionalización, implicando una mayor separación entre relaciones comunales y de asociación en términos weberianos. En segundo término, porque apoyándonos en ésta y otras investigaciones precedentes (Berti, 2006), es posible señalar que existen en este caso algunos cliques o grupos de empresas, particularmente en el sector electrónico, identificados por lazos fuertes que les han permitido realizar actividades de mayor compromiso.

Desde el punto de vista metodológico, se pueden elaborar también algunas conclusiones sobre los aportes y límites del ARS para el monitoreo y evaluación de estas políticas. 
Se debe comenzar por decir que a partir de los indicadores y variables presentados es posible comprender a nivel de la "red social" total en qué situación se halla una trama de empresas, detectar los niveles de conocimiento, de comunicación, de acción colectiva, de existencia de conflictos u otros aspectos que afectan la sustentabilidad de las redes. En segundo lugar, en el nivel de las "redes individuales" (ego networks) que en este artículo no se han profundizado, la metodología utilizada permite observar cómo son las relaciones y posiciones de cada una de las empresas, así como la existencia de subgrupos o empresas aisladas. De este modo, lo que en estudios habituales se reduce a variables generales y poco específicas como el concepto de "confianza" puede ser desagregado así en diversas dimensiones, permitiendo realizar un mejor diagnóstico y mejorar la gestión de la red. Incluso, esta información puede ser utilizada para realizar acciones de asistencia técnica basadas en la "administración de las redes" (network management), una competencia que ha podido ser estudiada y desarrollada a partir de la posibilidad de este tipo de análisis.

Estos indicadores pueden ser utilizados como parte de una "línea de base" y monitorearse a lo largo de la vida de un Programa o iniciativa de gestión, hasta su evaluación final para conocer cómo ha evolucionado la trama asociativa. Los indicadores generados pueden compararse además con otros encadenamientos, del mismo sector o de otros, lo que permitirá observar las diferencias de su estructura social y acumular evidencias sobre los valores y niveles de relación empíricamente alcanzados.

Estas potencialidades no niegan las limitaciones internas de la metodología (Miceli, 2008) que pueden resumirse según estén referidas al relevamiento y tipología de los datos o a su interpretación. En el primer caso, se destacan i) la tensión metodológica entre la necesidad de exhaustividad y el hecho de que ésta es prácticamente inviable ii) el necesario recorte del universo de análisis tanto a partir de datos atributivos como relacionales ocasionado por las limitaciones de accesibilidad pero también por los intereses del investigador (la construcción de redes incluye una cierta simplificación de las relaciones existentes y una exclusión de otras relaciones) iii) las dificultades para obtener respuesta de los empresarios, y la particular dificultad de los cuestionarios que incluyen generadores de nombres y largos listados iv) la clara dicotomía entre redes percibidas y redes observadas y la necesidad de tomar decisiones al respecto (algo que se puede ver por ejemplo en las alternativas para construir las relaciones de cooperación) v) también debe tenerse en cuenta la complejidad del ARS que conlleva costos para el trabajo de campo y el procesamiento de datos que deben ser analizados adecuadamente. 
En lo referido a la interpretación de los datos se puede señalar por su parte que: i) el potencial descriptivo a partir de la formalización de datos relacionales es relevante en forma numérica para redes de gran tamaño pero el potencial gráfico pierde mucho de su valor en ese contexto ii) probablemente por tratarse de un campo en pleno desarrollo, toda la capacidad descriptiva del ARS aún no ha llegado al mismo nivel en términos explicativos, en particular cuando se trabaja con datos dicotómicos. Esta situación hace que el paso de la descripción de las relaciones a la causalidad relacional no esté exenta de problemas y exige la interpretación del investigador iii) por cierto el ARS brinda muchas más posibilidades que las aquí utilizadas aunque en la mayor parte de los casos esto se debe a que los modelos teóricos subyacentes no son aplicables al fenómeno de la cooperación entre empresas. Asimismo se debe señalar que más allá de los resultados aportados por el ARS, éstos deben ser complementados por otros indicadores relacionados con las características y atributos de las empresas y del contexto. Como se ha visto en este estudio existen incluso diferencias al realizar un análisis (con los mismos datos) en términos de relaciones o de empresas iv) finalmente, si se pretende interpretar los datos aportados por el ARS en términos normativos, es preciso para ello contar con mayor cantidad de estudios que permitan generar análisis comparativos. Existe una tendencia a creer que valores altos de grado o densidad de una red son sinónimos de "bondad" de una red, y -al revés-, que la ausencia de estos valores indica la necesidad de promoverlos. En realidad, estudios como el aquí presentado han presentado algunas interesantes correlaciones entre "capital social" y "resultados económicos" pero si se quieren generalizar estas conclusiones se debe contar con estudios en redes similares que permitan analizarlas en contexto.

No obstante las limitaciones, estas mediciones constituyen un aporte en el marco de un proceso destinado a entender cómo las estructuras sociales pueden promover o impedir el buen desempeño económico de las firmas que trabajan de manera conjunta. Este es un punto de convergencia entre la mirada sociológica de las relaciones y los resultados económicos de este tipo de programas. La acción económica se encuentra sumergida en procesos sociales que facilitan o impiden su desarrollo, pero de manera frecuente éstos quedan vagamente definidos, por lo que pierden fuerza explicativa. La definición operativa de las relaciones interempresariales en las dimensiones reticulares aquí propuestas permitirán explorar cómo estas estructuras impulsan el crecimiento de las firmas individuales y particularmente de las formas en las que estas se estructuran, particularmente a nivel territorial. 


\section{Bibliografía}

ADLER, P.; KWON, S., (2002). "Social Capital: Prospects for a new concept." Academy of Management Review, 27(1): 17-40.

Aldrich, H., Zimmer, C., (1986), "Entrepreneurship through Social Networks", en Sexton, D. L., Smilor, R., (eds.) The Art and Science of Entrepreneurship, Cambridge.

Ariño, Africa; De la Torre, José; Smith Ring, Peter (2001). "Relational Quality: Managing Trust in Corporate Alliances," California Management Review 44/1: 109131.

Baranger, D., (2004). Epistemología y metodología en la obra de Pierre Bourdieu. Prometeo.Buenos Aires.

Baum, J.A.C., Calabrese, T., \& Silverman, B.S. (2000). "Don't go it alone: alliance network composition and start-up's performance in Canadian Biotechnology", Strategic Management Journal, 21: 267-294.

Berti, Natalia, (2006). «Del Combinado al Satélite. Trayectorias, Redes y Estrategias Empresariales del Complejo Electrónico Cordobés ». Tesis. Universidad Nacional de La Plata. Mimeo.

Birley, S., (1990). Entrepreneurs Networks: Their Creation and Development in Different Countries, Cranfield: Cranfield School of Management.

Bourdieu, P. (2001). Las estructuras sociales de la economía. Manantial, Bs As.

Bourdieu, Pierre. (1980). «Le capital social», en: Actes de la Recherche en Sciences Sociales, No. 31, pág. 2-3.

Brandenburger, A.M. \& Nalebuff, B.J. (1996). Co-opetition. New York: Doubleday.

Burt, R. (2000). The network structure of social capital. University of Chicago, Intitute Europeen d'Administration d' Affaires (INSEAD).

Burt, R., (1992). Structural holes. The social structure of competition. Harvard University Press, Cambridge.

Casanueva Rocha, Cristóbal (2003). «Relaciones estratégicas entre pymes: contraste de hipótesis empresariales mediante ARS ». Revista REDES.Vol.4,\#4 http://revista-redes.rediris.es-.

Castells, M. (1996). La era de la información: Economía, sociedad y cultura. Volumen 1: "La sociedad red", SXXI Editores. 
Castro, I. (2005). El capital social en las redes interorganizativas: Un estudio en el sector español de la construcción. Tesis Doctoral. Univ. de Sevilla.

Coleman, J. (1990). Foundations of Social Theory. Cambridge, Mass.: Harvard University Press.

Contractor, F.J. \& Lorange, P. (1988). "Why should firms cooperate? The strategy and economic basis for cooperative ventures". En Contractor y Lorange (Eds.), Cooperative Strategies in International Business. Lexington Books. Lexington.

Cross, Rob; Borgatti, Ph.; Parker, Andrew (2002). "Making invisible work visible: using social network analysis to support strategic collaboration".California Management review vol. 44,no. 2 winter.

Denzin, N. K. (1970): Sociological Methods: a Source Book. Aldine Publishing Company. Chicago.

Doz, Y.L. 1996. "The evolution of cooperation in strategic alliances: Initial conditions, or learning processes?" Strategic Management Journal, Summer Special Issue, 17: 55-83.

Etchegorry, C.; Magnano,C.; Matta, A., (2009). "Política pública y economía social: entre el parche y la fisura". Revista Administración Pública y Sociedad. No 16, pgs. 115-134.

Gnyawali, D.R. \& Madhavan, R. (2001). "Cooperative networks and competitive dynamics: A structural embeddedness perspective". Academy of Management Review, 26(3): 431-445.

Granovetter, M. (1973). "The Strenhgt of weak ties", American Journal of Sociology No 78.

Granovetter, M. (1995). "The Economic Sociology of Firms and Entrepreneurs." En Alejandro Portes, ed., The Economic Sociology of Immigration: Essays on Networks, Ethnicity, and Entrepreneurship. New York: Russell Sage Foundation.

Hakansson, L., Johanson,J., (1993), "The network as a governance structure" en G. Grabher (ed.), The Embedded Firm: the Socioeconomics of Industrial Networks. London: Routledge. 35-52.

Jensen, M., and Meckling, W. (1976). "Theory of the firm: Managerial behavior, agency costs, and ownership structure". Journal of Financial Economics, 3:305-360. Johannison, B. (2000). "Networking and entrepreneurial growth" en Sexton, D y Landstrom, H (Eds) Handbook of Entrepreneurship. London: Blackwell. 
Kale, P., Singh, H. y Perlmutter, H. (2000) "Learning and Protection of Proprietary Assets in Strategic Alliances: Building Relational Capital". Strategic Management Journal, Volumen 21, Número 3.

Labianca, G., Brass, D. J., \& Gray, B. 1998. Social networks and perceptions of intergroup conflict: The role of negative relationships and third parties. Academy of Management Journal, 41: 55-67.

Lado, A.A., Boyd, N. \& Hanlon, S.C. (1997). "Competition, cooperation, and the search for economic rents: A syncretic model". Academy of Management Review, 22(1): 110-141.

Lee, C., Lee K. y Pennings J (2001). "Internal capabilities, external networks, and performance: A study on technology-based ventures." Strategic Management Journal 22(6-7):615-640.

Laumann, E.; Marsden,P.; Prensky, D. (1983) "The Boundary Specification Problem in Network Analysis », en Burt R. y Minor M. (eds.), Applied Network Analysis. A Methodological Introduction, Beverly Hills: Sage, pp. 18-34.

Lin, N. (1999) "Building a network theory of social capital." Connections 22: 28-51. Matta, A. (2012) Redes, capital social y cooperación en el campo económico. Una aplicación del modelo de Análisis de Redes Sociales a la gestión de estrategias inter-organizacionales. Tesis de Doctorado en Ciencias Económicas. Universidad Nacional de Córdoba.Mimeo.

McCarty, Christopher; Molina, José Luis; Aguilar, Claudia y Laura Rota (2007). "A Comparison of Social Network Mapping and Personal Network Visualization", Field Methods, Vol. 19 (2) May (145-162).

McVay, M. (2000). "Midiendo el desempeño de los SDE: un alcance sumario". Revista "Desarrollo de la pequeña empresa". Intermediate Technoloy Publications.

Miceli, Jorge (2008). "Los problemas de validez en el análisis de redes sociales: Algunas reflexiones integradoras". Revista REDES Vol.14,\#1, Junio. http://revistaredes.rediris.es.

Milward, Brinton and PROVAN, Keith (1998). "Measuring network structure". Public administration.Vol76 summer 1998 387-407.Blackwell Publishers Oxford.

Mohr, J., Spekman, R. (1994). "Characteristics of partnership success: partnership attributes, communication behavior, and conflict resolution techniques", Strategic Management Journal, Vol. 15 No.2. 
Molina, José Luis (2005). "El estudio de las redes personales:contribuciones, métodos y perspectivas", Empiria, Julio-Diciembre 10 (71-106).

Parker, Andrew, Cross, Rob,Walsh, Dean (2001). "Improving collaboration with social network analysis Leveraging knowledge in the informal organization". Knowledge management review.Volumen 4 issue 2 may/jun.

Pelled, L. H., Eisenhardt, K. M., \& Xin, K. R. 1999. Exploring the black box: An analysis of work group diversity,conflict, and performance. Administrative Science Quarterly, 44: 1-28.

Pietrobelli, C, Rabelotti, R. (2006). Upgrading to Compete. Global Value Chains, Clusters, and SMEs in Latin America. Inter-American Development Bank. David Rockefeller Center for Latin American Studies. Harvard University.

Polanyi, K. (1947). La gran transformación. Ed Claridad. Bs As.

Premaratne, S. (2002). Entrepreneurial Networks and Small Business Development:The Case of Small Enterprises in Sri Lanka. Eindhoven: Technische Universiteit Eindhoven.Proefschrift.

Rabellotti, R. y Schmitz, H. (1999):"The internal heterogeneity of industrial districts in Italy, Brazil and Mexico", Regional Studies, vol. 33, núm. 2, pp. 97-108.

Scott, J. (2000). Social Network Analysis: A Handbook. 2nd Ed. Newberry Park, CA: Sage.

Simpson, William B. (2001) "QAP: The Quadratic Assignment Procedure." North American Stata Users' Group Meeting.

Sivadas Eugene Dwyer F. Robert (1998). "A Comparison of Organizational Factors Influencing New Product Success in Internal and Alliance Based Products" Report 4Institute for the Study of Business Markets The Pennsylvania State University.

Snijders, T.; Van de Bunt, G; Steglich, C. (2010) Introduction to stochastic actorbased models for network dynamics. Social Networks, 32.

Szarka, Joseph. (1990). "Networking and Small Firms." International Small Business Journal 8(2):10-22.

Tsai, W. (2000). 'Social capital, strategic relatedness and the formation of interorganizational linkages', Strategic Management Journal, 21, 925-939.

Tuomela, R., (2000). Cooperation: A Philosophical Study, Philosophical Studies Series 82, Kluwer Academic Publishers, Dordrecht 
Uzzi, B. (1997). "Social structure and competition in inter-firm networks: The paradox of embeddedness".Administrative Science Quarterly, 42: 35-67.

Veciana, J.M., Clarke, A. M., (1996). "Theoretical Approaches to Entrepreneurship: The Social Network Approach", European Doctoral Programme in Entrepreneurship and Small Business Management, University of Autónoma, Barcelona.

Williamson, O.E. (1995) Hierarchies, Markets and Power in the Economy: An economic Perspective.Industrial an Corporate Change, Volume 4, No1. Oxford University Press. 